Rapid Reviews COVID-19

\title{
Review 1: "Longitudinal monitoring of SARS-CoV-2 RNA on high-touch surfaces in a community setting"
}

Eric Meyerowitz ${ }^{1}$

${ }^{1}$ Montefiore Medical Center, Infectious Diseases, USA

Published on: Jan 31, 2021

DOI: 10.1162/2e3983f5.7b439401

License: Creative Commons Attribution 4.0 International License (CC-BY 4.0). 


\section{$\underline{\text { RR:C19 Evidence Scale rating by reviewer: }}$}

- Reliable. The main study claims are generally justified by its methods and data. The results and conclusions are likely to be similar to the hypothetical ideal study. There are some minor caveats or limitations, but they would/do not change the major claims of the study. The study provides sufficient strength of evidence on its own that its main claims should be considered actionable, with some room for future revision.

\section{Review:}

This is a fascinating paper in which the authors performed environmental surveillance of surfaces in Sommerville, Massachusetts from March through June 2020. They took swabs of 33 high touch surfaces from 12 locations during the study period. Surfaces including crosswalk buttons, a garbage can handle, door handles at a subway station, liquor store, convenience store, gas pump handles, and ATM keypads. They then extracted and amplified SARS-CoV-2 RNA from the swabs. Surfaces were swabbed weekly during the study period.

They found 29 out of 348 surface samples were positive at some point during the study, though importantly they found low amounts of RNA in the samples. More than half of the surfaces were positive at least one time during the study period. They found peaks in surface positivity preceded peaks in COVID-19 cases in the same zip code.

The authors note that their findings suggest that respiratory transmission is likely to be far more important than fomite transmission, which is supported by vast clinical and public health experience to date. They note that the fact that the surface positivity preceded peaks of local clinical cases suggests that environmental sampling might be a useful tool to predict outbreaks and could inform related public health measures.

In summary, the claims are well supported by the data and methods used, though sample sizes are small. The findings they present are actionable in that they suggest a framework for community monitoring that should be explored and studied more widely. The data on environmental sample positivity and implications for low risk of fomite transmission fits well with our current understanding of SARS-CoV-2 spread. The manuscript should be accepted with minor revisions only. I would recommend restructuring the Discussion section. Could consider the following outline: Paragraph 1: restate results without interpretation (\# positive surfaces, low RNA values, $>1 / 2$ of surfaces positive at some point, surfaces positive prior to local increase in covid cases). Paragraph 2: frame how this fits with our current understanding of transmission (quite well, even when surfaces positive, they have 
low RNA). Results very consistent with findings in prior studies. Cite the studies you mention and can look for potential additional studies in Table 1 of https://doi.org/10.7326/M20-5008. Totally agree with your point about importance of focusing on respiratory transmission primarily. Also, the comparisons with other viruses is excellent. Paragraph 3: the hypothesis generating novel finding is that the study found that surface positivity precedes case positivity. Your discussion is excellent on this point and the limitations of wastewater monitoring but would make clear that this needs to be studied further. Paragraph 4: discuss your limitations, including small study in a single setting. 